This item was submitted to Loughborough's Research Repository by the author.

Items in Figshare are protected by copyright, with all rights reserved, unless otherwise indicated.

\title{
Shape reconstruction using instruction systolic array
}

PLEASE CITE THE PUBLISHED VERSION

https://doi.org/10.1109/ICSENS.2017.8233988

PUBLISHER

(c) IEEE

VERSION

AM (Accepted Manuscript)

LICENCE

CC BY-NC-ND 4.0

REPOSITORY RECORD

Kandaswamy, Partheepan, James A. Flint, and V.A. Chouliaras. 2019. "Shape Reconstruction Using Instruction Systolic Array”. figshare. https://hdl.handle.net/2134/26508. 


\title{
Shape Reconstruction using Instruction Systolic Array
}

\author{
Partheepan Kandaswamy, James A Flint, Vassilios A Choularas \\ Wolfson School of Mechanical, Electrical and Manufacturing Engineering \\ Loughborough University \\ Loughborough, LE11 3TU \\ UK \\ p.kandaswamy@lboro.ac.uk
}

\begin{abstract}
This paper describes a novel, 2D mesh architecture prototype based on the Instruction Systolic Array (ISA) paradigm for distributed computing on fabrics. We discuss a real-time shape sensing and reconstruction application executing on this architecture and demonstrate a physical design for a wearable system based on ISA concept constructed out of off-the-shelf microcontrollers and sensors. Results demonstrate the application executes in $39 \mathrm{~ms}$ on our prototype ISA implementation thus confirming the viability of the proposed architecture for fabric-resident computing devices.
\end{abstract}

Keywords-Instruction Systolic Array; distributed sensor networks; System on fabrics; wearable electronics; fabric-resident computing device.

\section{INTRODUCTION}

The Instruction Systolic Array (ISA) was first introduced by Kung et al. in 1978 [1]. The ISA is a network which is composed of a large number of identical, locally connected elementary Processing elements (P). The ISA is a parallel computer model, an architectural concept suited for implementing a system with high bandwidth and architectural benefits for wearable applications. To implement the on-fabric ISA concept, shape reconstruction application has been chosen from Hermanis et al. [2]. The method is based on three-axial acceleration and magnetic sensor nodes that are embedded into the fabric and can measure local orientation data. The shape reconstruction algorithm along with ISA from local orientation measurements ensures fast computations for global shape reconstructions utilising data from a number of sensors.

\section{BACKGROUND}

ISA is broadly used in very-large-scale integration technology (VLSI) for execution purposes as an architectural concept [3] [4]. ISA is more flexible and advanced as compared to data systolic arrays which are considered chiefly as special purpose architectures. The main focus of ISA is on short interconnections for data communication as well as for control communication. The key properties of ISA are local communication for data and control flow, modularity and scalability, local data handling and mapping is logical.

In ISA, rather than data, instructions and selector bits are pumped in a systolic way through a processor array. This particular arrangement helps in executing different algorithms on the same processor array. Also, the instruction stream and the selector bit stream get combined for the execution of ISA as shown in Fig. 1. To execute the instructions at a particular processing element, the selector bit must be 1 . The fundamental model of a parallel computer can be seen as a mesh connected $\mathrm{n} \times \mathrm{n}$-array with identical processors. The processors are capable of executing instructions from a small instruction set. Instructions, as well as selector bits, are used for controlling processing elements. The flow of instructions is generally from top to bottom (north to south) of the array. On the other hand, the selector bit flows from left to right (west to east) of the array.

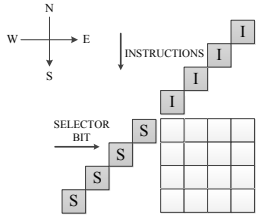

(1)

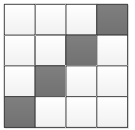

(5)

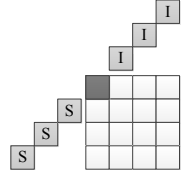

(2)

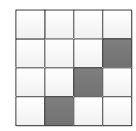

(6)

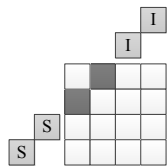

(3)

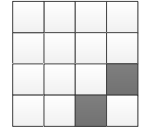

(7)

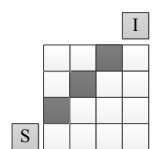

(4)

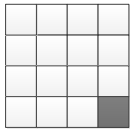

(8)
Fig 1: Execution of an ISA

\section{NOVEL ARCHITECTURE FOR ON-FABRIC PARALLEL PROCESSING}

The processing elements are arranged in a systolic manner as shown in Fig. 2. Each processing element is connected to their neighbouring processing elements and also closely coupled to the sensors. Due to large number of physical interconnections that are likely to be necessary to wire a suitable-sized sensor array, a compromise has to be taken by selecting the $\mathrm{I} 2 \mathrm{C}$ bus and sharing it for both the ISA inter element connections and sensors. Each processing element has 
four $\mathrm{I} 2 \mathrm{C}$ buses, one per direction. The north and west ports act as slaves and the east and south ports are the master devices. The northern boundary of the array is connected to the instruction stream flow controller (the external host) which stores the array of instructions that needs to be passed to the processing elements. The western boundary of the array is connected to selector bit flow controller (also the external host) which stores the array of selector bits that needs to be passed to the processing elements. The processing elements and the sensors are closely coupled as shown in Fig. 2 so that processing can be done locally.

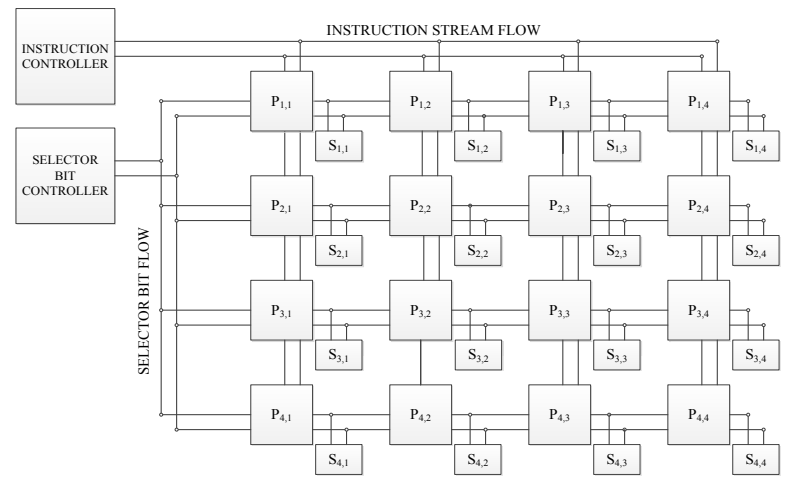

Fig 2: System Architecture-Processor Array (P-Processing element, SSensor)

\section{SHAPE RECONSTRUCTION ALGORITHM}

As acceleration/magnetic sensors provide only two vector observations, which is minimum for full orientation determination, no real minimisation problem can be defined. Therefore, Hermanis et al. proposed the triad based computation for fast, singularity free and a computationally simple algorithm. They constructed two triads of orthonormal unit vectors, one formed from the general reference frame, the other from the sensor reference frame. Equations of the triads are referenced from [2]. These triads are then used to form the global earth reference and sensor measurement matrices. The authors then compute the rotation matrix which describes sensor orientation relative to the global reference frame. The rotational matrix is used to calculate the surface segment orientation relative to initial position that corresponds to sensor orientation relative to earth reference frame. Acceleration and magnetic sensor nodes are arranged in regular grid along the surface. The model of the surface is divided in $\mathrm{n}$ rigid segments. Each segment is described by four direction vectors. From the segment structure it can be deduced, that if a single control point location is known, then any other control point on the same segment row or column can be calculated by adding and subtracting the corresponding segment direction vectors.

\section{EXPERIMENTAL SETUP}

A concept prototype of the ISA concept was designed using commercially available ARM Cortex-M0+ LPC824 microcontrollers as processing elements, and instruction and selector bit flow controllers shown in Fig. 3 to demonstrate the feasibility of our system, validate its applicability on a wearable-computing application, estimate its performance on the latter and propose ways of improving it.

To implement the surface reconstruction application using ISA, a sensor network with 16 sensors was embedded in a piece of fabric. Inter-node communication is via I2C, allowing the connection of sensors using four wires. A sensor node built with the LSM303DLHC acceleration/magnetic sensor is shown in Fig. 3. The LSM303DLHC is used for orientation estimation as described in section IV. Each sensor is connected to its own microcontroller as shown in the system architecture of Fig. 2. The microcontroller serves as the interface between the sensor node and the host computer as all the computations take place locally in the microcontrollers. When fully implemented the umbilical shown in Fig. 3 will not exist. Each microcontroller is assigned a unique ID to identify its position in the grid and calculate the control points. Once the microcontroller receives the ID, it starts to receive the orientation data from the sensor. The orientation data is then averaged and stored for calculation of directional vectors. These directional vectors are shared between neighboring microcontrollers for the calculation of control points. Once these control points are calculated for each sensor, they are sent to the host computer via serial port for $3 \mathrm{D}$ visualisation of the sensed object. The process of ISA computing the control points and the host drawing the visualisation continues indefinitely.

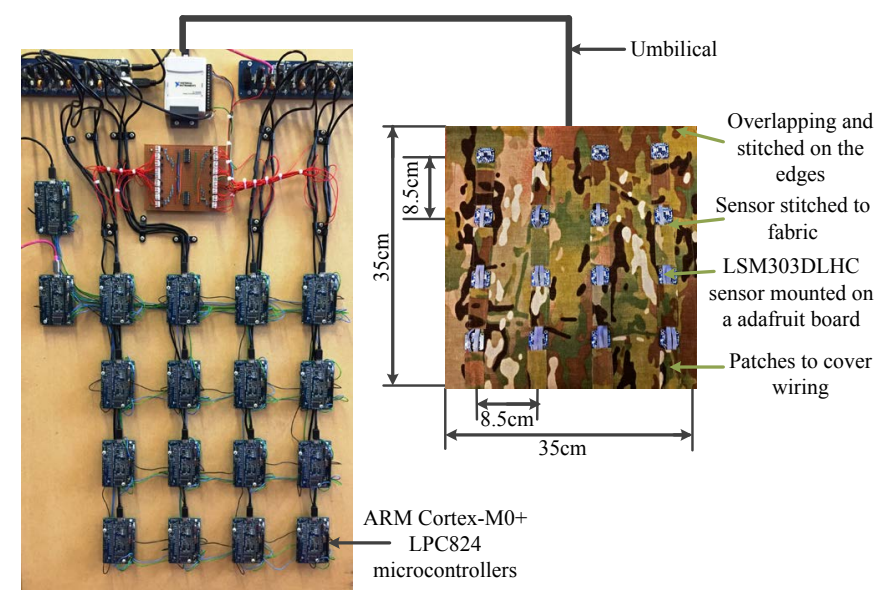

Fig 3: Prototype board (left) and Sensors embedded in fabric (cm-centimeter) (right)

\section{PROGRAMMING THE ISA}

The ISA application is programmed on to the chosen microcontrollers. All the microcontrollers will be having the same program flashed in them. All microcontrollers are aware of their position and at the boundary sending of instruction and selector bits is disabled as the end of the array has been reached. The working of the microcontroller program is explained in Fig 4. The instruction and selector bits are passed to the processing element; the latter is in the listening mode waiting for the frame bytes to be received from the north and west slave $\mathrm{I} 2 \mathrm{C}$ ports. Once both the instruction and selector bit are received they are then decoded and executed through an 
interrupt service routine. After the execution the instruction and the selector bit are then forwarded to the neighbors through the south and east master I2C ports. A diagonal of instruction and their corresponding selector bit that is used for implementing the shape reconstruction application is shown in Fig. 4. Set of no operation is flushed through the array before and after the instruction and selector bit diagonal.
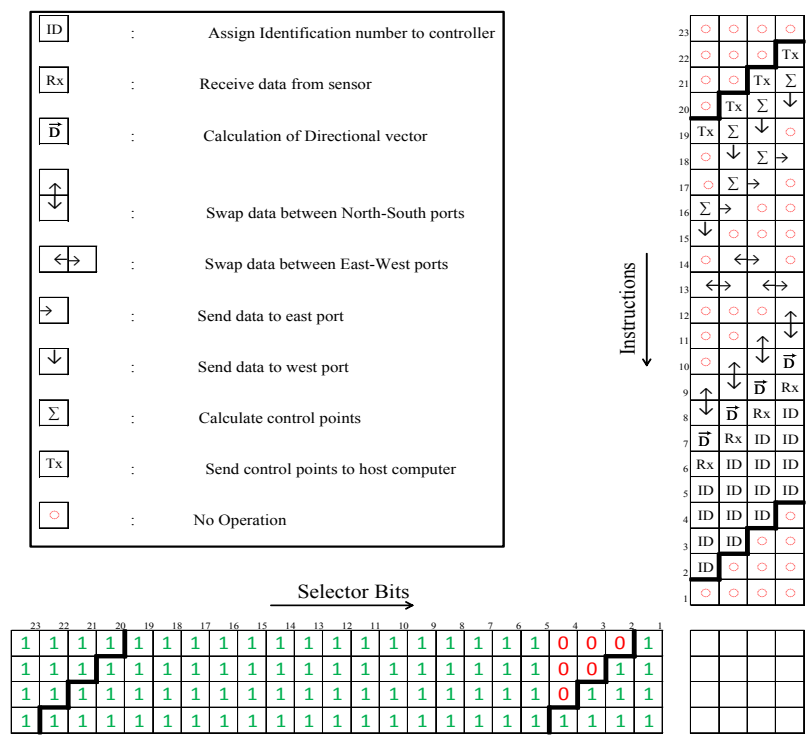

Fig 4: A diagonal of instruction and corresponding selector bit for the shape reconstruction application and the illustration of the instructions

\section{RESULTS}

A network of 16 sensor nodes was experimentally tested. Sensors were arranged on a $4 \times 4$ grid sewed on the layer of fabric with mutual distances $8.5 \mathrm{~cm}$ long ways and $8.5 \mathrm{~cm}$ across as shown in Fig. 3.

To evaluate the accuracy of proposed shape sensing method, two experiments were performed. The first involved the fabric around a cylindrical object placing the object vertically and then reconstructing its shape as shown in figure 5(a) and the reconstructed image is shown in $5(\mathrm{~b})$.
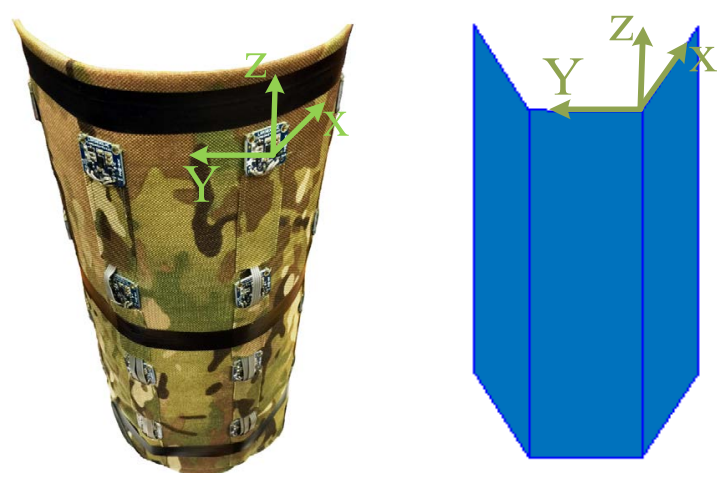

(a) (b)
Fig 5: (a) Fabric wrapped on a vertical object (b) Reconstructed shape

The graph Fig .6 is plotted against instruction/selector cycle vs time. It indicates the time difference between the instruction received and the selector bit sent between the processing elements. A few instructions take longer to execute because of their implementation complexity. For example the $6^{\text {th }}$ instruction on $\mathrm{P}(1,2)$ and the $7^{\text {th }}$ instruction on $\mathrm{P}(2,3)$ which is a sensor read takes few milliseconds to read from the sensor and to average and store it in the register for further computation. Delays occur also because of $\mathrm{I} 2 \mathrm{C}$ bus communication.

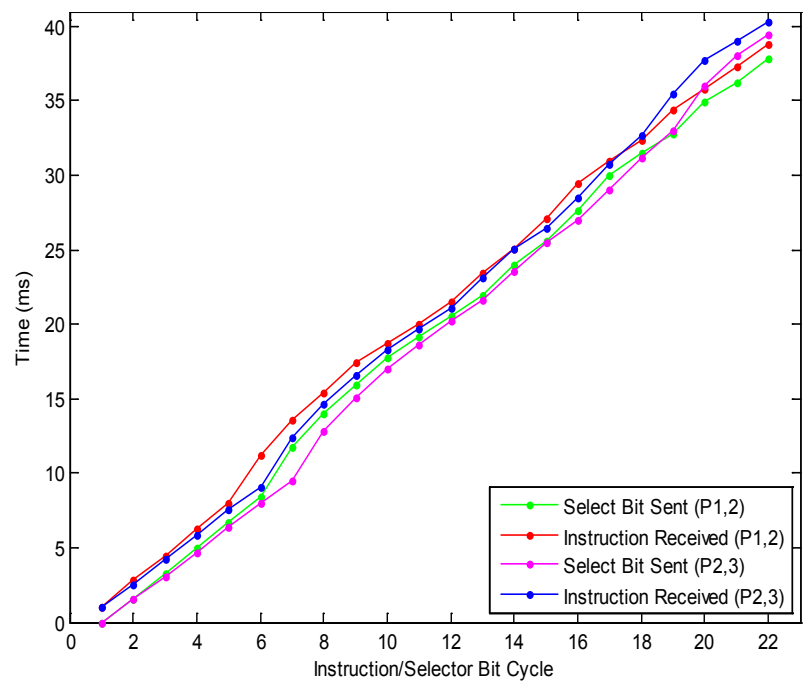

Fig 6: Performance analysis for $\mathrm{P}(1,2)$ and $\mathrm{P}(2,3)$

\section{CONCLUSION}

The wearable shape reconstruction application has been successfully implemented using our proposed concept of ISA architecture constructed out of off-the-shelf microcontrollers and sensors. Results demonstrate the application executes in $39 \mathrm{~ms}$ on our prototype ISA implementation thus confirming the viability of the proposed architecture for fabric-resident computing devices. Future work will focus on extending the supported instructions, optimising the I2C medium and allowing for more concurrency, at node level, between computation and communication.

\section{REFERENCES}

[1] H. T. Kung, C. E. Leiserson, Systolic Arrays (for VLSI), Technical Report CS 79-103, Carnegie Mellon University, 1978.

[2] A. Hermanis, R. Cacurs, M. Greitans, "Acceleration and magnetic sensor network for shape sensing," IEEE Sensors, vol. 16, no. 5, pp. 1271-1280, March 2016.

[3] M. Kunde, H.W. Lang, M. Schimmler, H. Schmeck, and H. Schroder, "The instruction systolic array and its relation to other models of parallel computers," Parallel Computing, vol. 7, pp. 25-39, 1988.

[4] H.W. Lang, "The instruction systolic array - a parallel architecture for VLSI,” Integration, vol. 4, pp. 65-74, 1986. 